\title{
HZD-Based Control of a Five-Link Underactuated 3D Bipedal Robot
}

\author{
J.W. Grizzle, Christine Chevallereau, and Ching-Long Shih
}

\begin{abstract}
This paper presents a within-stride feedback controller that achieves an exponentially stable, periodic, and fast walking gait for a 3D bipedal robot consisting of a torso, revolute knees, and passive (unactuated) point feet. The walking surface is assumed to be rigid and flat; the contact between the robot and the walking surface is assumed to inhibit yaw rotation. The studied robot has $8 \mathrm{DOF}$ in the single support phase and 6 actuators. In addition to the reduced number of actuators, the interest of studying robots with point feet is that the feedback control solution must explicitly account for the robot's natural dynamics in order to achieve balance while walking. We use an extension of the method of virtual constraints and hybrid zero dynamics (HZD), a very successful method for planar bipeds, in order to determine a periodic orbit and an autonomous feedback controller that realizes the orbit, for a 3D (spatial) bipedal walking robot. The effect of output selection on the zero dynamics is highlighted and a pertinent choice of outputs is proposed, leading to stabilization without the use of a supplemental event-based controller.
\end{abstract}

\section{INTRODUCTION}

The primary objective of this paper is to demonstrate that the methods presented in [27] for underactuated planar bipedal robots (i.e., bipeds constrained to the sagittal plane of motion) have a natural extension to underactuated spatial (or 3D) bipedal robots. In particular, the work presented here addresses the frontal plane dynamics (i.e., side-to-side motion) in addition to the sagittal plane dynamics treated in [27]. We study a simple 5-link robot model with an unactuated point contact at the leg ends and seek a timeinvariant feedback controller that creates an exponentially stable, periodic walking motion.

Our control design is based on the method of virtual constraints and hybrid zero dynamics (HZD), which generalizes the fundamental work of Byrnes and Isidori [11] to the hybrid setting [26]. In our first attempt at applying these methods to a simple 3-link spatial model, we selected the actuated joints as the outputs for the purpose of designing the virtual constraints [19]. Optimization of the free parameters in the virtual constraints was used to compute a periodic orbit, but unlike the planar designs in [27], the orbit was unstable under the nominal continuous-time controller used to zero the outputs. An event-based controller was then designed,

Corresponding Author: Jessy W. Grizzle is with the Control Systems Laboratory, Electrical Engineering and Computer Science Department, University of Michigan, Ann Arbor, MI 48109-2122, USA, grizzle@umich.edu.

Christine Chevallereau is with the CNRS, IRCCyN, Nantes Atlantic University, 1 rue de la Noe, 44321 Nantes, cedex 03, France, Christine. Chevallereaulirccyn.ec-nantes.fr

Ching-Long Shih is with the EE Department, National Taiwan University of Science and Technology, Taipei, Taiwan 106, shihcl@mail.ntust.edu.tw which, in conjunction with the continuous-time controller, yielded exponential stability of the periodic orbit.

In the current paper, we provide a choice of outputs for feedback design that results in exponential stability of a periodic orbit without recourse to event-based control. The advantages of this include a simpler overall feedback design and an immediate reaction to perturbations instead of waiting for the next impact. In a journal submission under review for IEEE-TRO, we compare both controllers [3] (a pre-print is available at [8]).

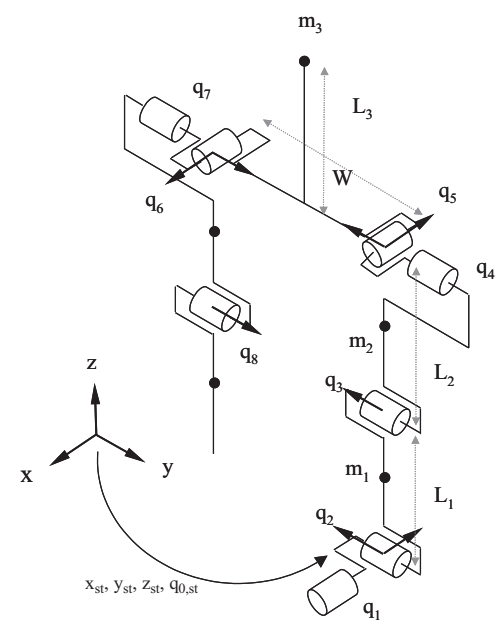

Fig. 1. A five-link 3D biped with point feet in support on leg-1. There is no yaw motion about the stance leg end and the degrees of freedom at the leg end specified by $q_{1}$ and $q_{2}$ are unactuated. The remaining joints are actuated. Consequently, in single support, the robot has $8 \mathrm{DOF}$ and 6 independent actuators. For simplicity, each link is modeled by a point mass at its center.

\section{RELATED WORK}

The work most closely related to ours is [6], where the control of a 3D walker was decomposed into the study of its motion in the sagittal plane and the frontal plane; see also [14] for a related decomposition result on control in the frontal plane. The method of virtual constraints was applied in [6] to regulate the sagittal plane motion of the biped, while an inverted pendulum approximation of the dynamics was used to design a controller for the frontal plane. An event-based controller was then introduced to synchronize the phasing of the independently designed sagittal and frontal plane controllers. The overall closed-loop system was shown to be stable through simulation and subsequently through experimentation. In our approach, we do not decompose the model into sagittal and frontal plane motions, and coupling of the sagittal and frontal plane dynamics is introduced into the controller from the very beginning. 
A very interesting study of the feedback control of underactuated spatial robots has been given in [20], where a controller for a five-link 3D robot with unactuated point feet has been designed on the basis of linearizing the robot's dynamic model along a periodic orbit. So that the controller would be time-invariant, the orbit was parameterized with a configuration variable that is strictly monotonic throughout a normal gait, as in [9], [18], [2], [26], before linearization was applied. The (within-stride) control law is designed on the basis of a discrete-time approximation of the linearized model, which makes stability of the closed-loop system difficult to assess.

Other important work includes [5] and references therein, where the analysis of passive spatial bipeds is presented. The emphasis in their work is on energy efficiency and underactuation; the role of feedback control in achieving a wide range of behaviors is not emphasized. On the other hand, the work in [22], [1] seeks energy efficiency and a large basin of attraction under the assumption of full actuation; in particular, full actuation between the leg and ground is assumed (pitch, roll and yaw), as opposed to the unactuated assumption made here. Very careful stability analysis of the closed-loop system is provided through geometric (Routhian) reduction. This work is taken one step further in [7], where, starting from a 2D (sagittal plane) passive limit cycle, the authors use geometric reduction to first achieve control of the frontal plane motion and then a second stage of geometric reduction to achieve steering within the walking surface.

To the best of our knowledge, other work on the control of spatial robots either assumes full actuation or does not provide significant analysis of the closed-loop system. There are many control strategies based on the zero moment point ZMP [24], with one of the more famous users being the robot ASIMO [23]. In this approach, a desired trajectory of the ZMP is defined and successive inner control loops are closed on the basis of the ZMP. In the work of [13], predictive control is performed on the basis of the position of the center of mass and a simplified model of the robot in order to achieve a desired ZMP trajectory. Recently, on-line adjustment of the ZMP has been added [12]; this control method is implemented on the robot HRP2. The control of the ZMP ensures that the supporting foot will not rotate about its extremities, but this does not ensure stability in the sense of convergence toward a periodic motion, as proved in [4].

\section{MODEL}

The model presented here was chosen to be complex enough to capture interesting features of gait control that do not occur in planar robots, and simple enough that the presentation of the ideas will remain transparent. It is our expectation that the ideas presented here apply to a wider class of bipeds, but proving such a conjecture is not an objective of this paper.

\section{A. Description of the robot and the walking gait}

The 3D bipedal robot discussed in this work is depicted in Fig. 1. It consists of five links: a torso and two legs with revolute one DOF knees that are independently actuated and terminated with "point-feet". Each hip consists of a revolute joint with two degrees of freedom and each degree of freedom (DOF) is independently actuated. The width of the hips is nonzero. The stance leg is assumed to act as a passive pivot in the sagittal and frontal planes, with no rotation about the z-axis (i.e., no yaw motion), so the leg end is modeled as a point contact with two DOF and no actuation. This model corresponds to the limiting case of robot with feet when the size of the feet decreases to zero. The unactuated DOF at the leg ends correspond to the classical DOF of an ankle. The DOF corresponding to the swing-leg ankle are not modelled. In total, the biped in the single support phase has eight DOF, and there are two degrees of underactuation.

The following assumptions are made in this study:

- Each link is rigid and has mass.

- Walking consists of two alternating phases of motion: single support and double support.

- The double support phase is instantaneous and occurs when the swing leg impacts the ground.

- At impact, the swing leg neither slips nor rebounds.

- The swing and stance legs exchange their roles at each impact.

- The gait is symmetric in steady state.

- Walking takes place on a flat surface.

A more detailed list of hypotheses is given in [27, Chap. 3] for planar robots, and with the obvious modifications for spatial robots, those hypotheses apply equally well here.

Since the gait is composed primarily of single support phases, the variables used to describe the robot are adapted to this phase of motion. The robot is represented as a tree structure. The stance foot, which is fixed on the ground, is the base of the tree structure. A set of generalized coordinates $q=\left[q_{1}, \ldots, q_{8}\right]^{\prime}$ is shown in Fig. 1. Absolute angles $\left(q_{1}, q_{2}\right)$ are roll and pitch angles of the stance leg, respectively. Angles $q_{3}$ and $q_{8}$ are the relative joint angles of the stance-leg knee and swing-leg knee, respectively. Angles $q_{4}$ and $q_{5}$ are the joint angles of the stance leg relative to the torso along the $y$-axis and the $x$-axis, respectively, and angles $q_{6}$ and $q_{7}$ are the joint angles of the swing leg relative to the torso along the $x$-axis and the $y$-axis, respectively. The coordinates $\left(q_{1}, q_{2}\right)$ are unactuated (due to the passive contact), while $\left(q_{3}, \ldots, q_{8}\right)$ are independently actuated.

The position of the robot with respect to an inertial frame is defined by adding the four variables $q_{e}=$ $\left[q^{\prime}, x_{s t}, y_{s t}, z_{s t}, q_{0, s t}\right]^{\prime}$, where $x_{s t}, y_{s t}$ and $z_{s t}$ are the Cartesian coordinates of the stance foot ${ }^{1}$, and $q_{0, s t}$ defines the rotation along the $\mathrm{z}$-axis of the stance leg. These variables are constant during each single support phase, but are discontinuous when the supporting leg changes (i.e., at each impact).

We have chosen to define the generalized coordinates with respect to the contact point of the current stance foot. When leg-2 is the supporting leg, the variables are defined as shown in Fig. 2 and the same notation is employed as when the supporting leg is leg-1, viz. Fig. 1. During each single

\footnotetext{
${ }^{1}$ The leg ends are referred to as feet or point feet.
} 
support phase, only one set of coordinates is used, depending on which leg is the supporting leg. In double support, either set of coordinates may be used. The transformation from one set of coordinates to the other is nonlinear [20], but it can be computed in closed form by standard means.

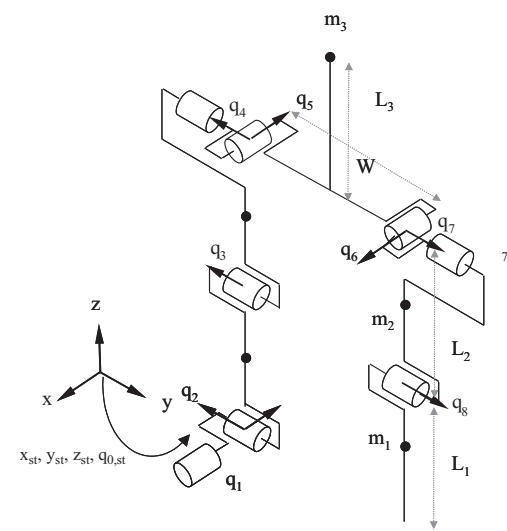

Fig. 2. A five-link 3D point-feet biped in support on leg-2. The DOF at the leg end (foot) are not actuated.

The legs exchange roles from one step to the next. If $T$ is the duration of a step, on a periodic walking cycle, due to the choice of coordinates in Figs. 1 and 2, we must have

$$
\begin{array}{ll}
q_{1}(t+T)=-q_{1}(t) & q_{2}(t+T)=q_{2}(t) \\
q_{3}(t+T)=q_{3}(t) & q_{4}(t+T)=q_{4}(t) \\
q_{5}(t+T)=-q_{5}(t) & q_{6}(t+T)=-q_{6}(t) \\
q_{7}(t+T)=q_{7}(t) & q_{8}(t+T)=q_{8}(t) \\
\text { and } & q_{0, s t}(t+T)=-q_{0, s t}(t) .
\end{array}
$$

The last condition yields a motion without yaw rotation.

\section{B. Dynamic model}

The dynamic models for single support and impact (i.e., double support) are derived here assuming support on leg 1 . The models for support on leg 2 can be written in a similar way. The Euler-Lagrange equations yield the dynamic model for the robot in the single support phase as

$$
D(q) \ddot{q}+H(q, \dot{q})=B u=\left[\begin{array}{c}
0_{2 \times 6} \\
I_{6 \times 6}
\end{array}\right] u,
$$

where $D(q)$ is the positive-definite $(8 \times 8)$ mass-inertia matrix, $H(q, \dot{q})$ is the $(8 \times 1)$ vector of Coriolis and gravity terms, $B$ is an $(8 \times 6)$ full-rank, constant matrix indicating whether a joint is actuated or not, and $u$ is the $(6 \times 1)$ vector of input torques. Following standard practice in the literature, the double support phase is assumed to be instantaneous. However, it actually consists of two distinct subphases: the impact, during which a rigid impact takes place between the swing foot and the ground, and coordinate relabeling. During the impact, the biped's configuration variables do not change, but the generalized velocities undergo a jump. The derivation of the impact model in double support phase requires the use of the vector $q_{e}$. Conservation of momentum during the impact process and the swing leg neither slipping

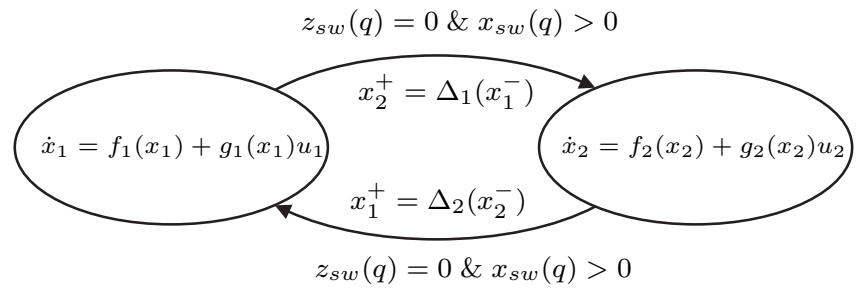

Fig. 3. Bipedal robot's dynamic model as a hybrid system.

nor rebounding at impact yield

$$
\left[\begin{array}{l}
\dot{q}_{e}^{+} \\
F_{s w}
\end{array}\right]=\left[\begin{array}{ll}
D_{e} & -E_{s w}^{\prime} \\
E_{s w} & 0_{4 \times 4}
\end{array}\right]^{-1}\left[\begin{array}{c}
D_{e} \dot{q}_{e}^{-} \\
0_{4 \times 1}
\end{array}\right]
$$

where $\dot{q}_{e}^{-}$and $\dot{q}_{e}^{+}$are the extended velocities before and after the impact, respectively, $F_{s w}$ is the reaction force at the contact point, $D_{e}$ is the extend mass-inertia matrix, and $E_{s w}=\frac{\partial}{\partial q_{e}}\left[x_{s w}, y_{s w}, z_{s w}, q_{0, s w}\right]^{\prime}$ is the Jacobian matrix for the position of the swing foot and its orientation in the $x-y$ plane. Analogously to [9], the overall impact model is written as

$$
\begin{aligned}
& q^{+}=\Delta_{q}\left(q^{-}\right) \\
& \dot{q}^{+}=\Delta_{\dot{q}}\left(q^{-}, \dot{q}^{-}\right),
\end{aligned}
$$

and is obtained from solving (3) and projecting down to the generalized coordinates for support on leg 2 .

Define state variables as $x_{j}=\left[\begin{array}{c}q \\ \dot{q}\end{array}\right]$, and let $x_{j}^{+}=$ $\left[\begin{array}{c}q^{+} \\ \dot{q}^{+}\end{array}\right]$and $x_{j}^{-}=\left[\begin{array}{c}q^{-} \\ \dot{q}^{-}\end{array}\right]$, where the subscript $j$ denotes the stance leg number. Then a complete walking motion of the robot can be expressed as a nonlinear system with impulse effects, as shown in Fig. 3 and written as

$$
\Sigma:\left\{\begin{array}{ll}
\dot{x}_{1}=f_{1}\left(x_{1}\right)+g_{1}\left(x_{1}\right) & u_{1} \quad x_{1}^{-} \notin S_{1} \\
x_{2}^{+}=\Delta_{1}\left(x_{1}^{-}\right) & x_{1}^{-} \in S_{1} \\
\dot{x}_{2}=f_{2}\left(x_{2}\right)+g_{2}\left(x_{2}\right) & u_{2} \quad x_{2}^{-} \notin S_{2} \\
x_{1}^{+}=\Delta_{2}\left(x_{2}^{-}\right) & x_{2}^{-} \in S_{2}
\end{array},\right.
$$

where $S_{j}=\left\{(q, \dot{q}) \mid z_{s w}(q)=0, \quad x_{s w}(q)>0\right\}$ is the switching surface, where the subscript $j=1,2$ denotes the stance leg number,

$f_{j}(x)=\left[\begin{array}{c}\dot{q} \\ -D^{-1}(q) H(q, \dot{q})\end{array}\right], \quad g_{j}(x)=\left[\begin{array}{c}0 \\ D^{-1}(q) B\end{array}\right]$,

and

$$
x_{k}^{+}=\Delta_{j}\left(x_{j}^{-}\right)=\left[\begin{array}{c}
\Delta_{q}\left(q^{-}\right) \\
\Delta_{\dot{q}}\left(q^{-}, \dot{q}^{-}\right)
\end{array}\right], k \neq j .
$$

\section{ViRTUAL CONSTRAINTS}

The method of virtual constraints, which has proven very successful in designing feedback controllers for stable walking in planar bipeds [9], [18], [2], [26], will be applied to the $3 \mathrm{D}$ biped of the previous section. In this method, one holonomic constraint per actuator is proposed in the form of an output that, when zeroed by a feedback controller, enforces the constraint. The most direct form of the constraint is [27, Chap. 6.4]

$$
y=h(q)=q_{a}-h_{d}(\theta)
$$


where $q_{a}=\left[q_{3}, q_{4}, q_{5}, q_{6}, q_{7}, q_{8}\right]^{\prime}$ is the 6-vector of actuated coordinates, $\theta=\theta(q)$ is a quantity that is strictly monotonic (i.e., strictly increasing or decreasing) along a typical walking gait, and $h_{d}(\theta)$ is the desired evolution of the actuated variables as a function of $\theta$. Roughly speaking, $\theta$ is used to replace time in parameterizing a periodic motion of the biped. In a forward walking motion, the speed of the biped is roughly proportional to the angle of the virtual stance leg in the sagittal plane, where the virtual stance leg is given by the line that connects the stance foot to the stance hip. When the shin and the thigh have the same length, the angle of the virtual leg is

$$
\theta=-q_{2}-q_{3} / 2
$$

(the minus sign is used to make $\theta$ strictly increasing over a step).

The outputs (7) are easily checked to have uniform relative degree 2 (i.e., relative degree two and an invertible decoupling matrix). The torque $u^{*}$ required to enforce the virtual constraint corresponding to $q_{a}=h_{d}(\theta)$ can be computed as ${ }^{2}$

$$
u^{*}=\left(\frac{\partial h(q)}{\partial q} D^{-1} B\right)^{-1}\left(\frac{\partial^{2} h_{d}(\theta)}{\partial \theta^{2}} \dot{\theta}^{2}(t)+\frac{\partial h(q)}{\partial q} D^{-1} H(q, \dot{q})\right)
$$

This leads to an input-output linearizing controller to asymptotically enforce the constraints [15] [27, Chap. 5],

$$
u=u^{*}-\left(\frac{\partial h}{\partial q} D^{-1} B\right)^{-1}\left(\frac{K_{p}}{\varepsilon^{2}} y+\frac{K_{d}}{\varepsilon} \dot{y}\right),
$$

which results in

$$
\ddot{y}+\frac{K_{d}}{\varepsilon} \dot{y}+\frac{K_{p}}{\varepsilon^{2}} y=0 .
$$

In other words, determining the constraints is equivalent to the design of a feedback controller in the single support phase, up to the choice of the gains $K_{p}>0, K_{d}>0$, and $\epsilon>0$ such that (11) is exponentially stable and converges sufficiently rapidly with respect to the duration of a single support phase; see [27, Chap. 4].

The swing phase zero dynamics is easily computed and has dimension 4 (the robot's model has dimension 16 (8 DOF) and the 6 outputs (7) have uniform relative degree 2). Let $q_{u}=\left[q_{1}, \theta\right]^{\prime}$ denote the unactuated joints and $q_{a}=$ $\left[q_{3}, q_{4}, q_{5}, q_{6}, q_{7}, q_{8}\right]^{\prime}$ denote the controlled joints, which are selected here to be the actuated joints. A linear relation exist between $q, q_{u}$ and $q_{a}$,

$$
q=\mathcal{T}\left[\begin{array}{l}
q_{u} \\
q_{a}
\end{array}\right]
$$

where $\mathcal{T}$ is an $(8 \times 8)$ invertible matrix. Then $(2)$ can be rewritten as

$$
\mathcal{T}^{\prime} D(q) \mathcal{T}\left[\begin{array}{c}
\ddot{q}_{u} \\
\ddot{q}_{a}
\end{array}\right]+\mathcal{T}^{\prime} H(q, \dot{q})=\mathcal{T}^{\prime} B u=\left[\begin{array}{c}
0_{2 \times 6} \\
I_{6 \times 6}
\end{array}\right] u
$$

The first two lines of the RHS of this equation are zero, yielding

$$
D_{11}(q) \ddot{q}_{u}+D_{12}(q) \ddot{q}_{a}+H_{1}(q, \dot{q})=0_{2 \times 1},
$$

\footnotetext{
${ }^{2}$ As shown in [21], [27, pp. 60], an expression for $u^{*}$ can be obtained without inverting of the $(8 \times 8)$ mass-inertia matrix $D$ is also possible.
}

where $D_{11}$ is the $(2 \times 2)$ upper left sub-matrix of $\mathcal{T}^{\prime} D(q) \mathcal{T}$, $D_{12}$ is the $(2 \times 6)$ upper right sub-matrix of $\mathcal{T}^{\prime} D(q) \mathcal{T}$ and $H_{1}(q, \dot{q})$ consists of the first two lines of $\mathcal{T}^{\prime} H(q, \dot{q})$. Substituting the expressions of $q_{a}, \dot{q}_{a}$ and $\ddot{q}_{a}$ corresponding to the virtual constraints, the dynamic model of the single support phase is now reduced to a low-dimensional, 2 DOF, autonomous system,

$$
\begin{gathered}
D_{11}\left(q_{u}\right)\left[\begin{array}{c}
\ddot{q}_{1} \\
\ddot{\theta}
\end{array}\right]+D_{12}\left(q_{u}\right)\left(\frac{\partial h_{d}}{\partial \theta} \ddot{\theta}+\frac{\partial^{2} h_{d}}{\partial \theta^{2}} \dot{\theta}^{2}\right) \\
+H_{1}\left(q_{u}, \dot{q}_{u}\right)=0,
\end{gathered}
$$

namely the swing phase zero dynamics [10], [27, Chap. 5].

One can clearly see that the dynamic properties of the swing phase zero dynamics depend on the particular choice of the virtual constraint $0=y=q_{a}-h_{d}(\theta)$. How to determine a choice for $h_{d}(\theta)$ that results in a periodic walking motion is summarized in the next section.

\section{Design for a Symmetric Periodic Gait}

The objective of this section is to design virtual constraints $q_{a}=h_{d}(\theta)$ that correspond to a periodic motion of the robot. The gait considered is composed of single support phases separated by impacts as described in Fig. 3. The legs exchange roles from one step to next, and due to symmetry, the study of a gait can be limited to a single step and the use of the symmetry relation (1).

\section{A. Virtual constraints and Bezier polynomials}

The problem of designing the virtual constraints will be transformed into a parameter optimization problem as in [27, Chap. 6]. Here, our main goal is to obtain a periodic motion; optimality is not so crucial. To simplify the optimization process, the number of variables used in the optimization problem is first reduced. This is accomplished by exploiting boundary conditions that arise from periodicity. Bezier polynomials are parametric functions that allow one to easily take into account boundary conditions on the configuration and velocity at the beginning and end of a step.

The initial and final configuration and velocity of the robot for a single support phase are important for defining the passage between the single and double support phases. Because the terminal configuration of the robot is chosen to be the instant before the double support configuration, both legs are in contact with the ground and therefore only seven independent variables are needed to describe this configuration (a closed kinematic chain). These variables parameterize the final configuration of the first step denoted $q_{f}$. The eight joint velocities $\dot{q}_{f}$ are independent and are also added.

Knowing the final state of the single support phase, the impact model (4) and (5) determines the initial state of the ensuing single support phase. The symmetry condition (1) then gives the initial state of the first step: $q_{i}, \dot{q}_{i}$. The initial orientation $\left(q_{i}\right)_{0, s t}$ of the robot is calculated such that the orientation for the second step is symmetric to the orientation for the first step in order that no yaw rotation is observed during the nominal (periodic) gait. 
To obtain a periodic gait, the single support must be such that the state of the robot evolves from $q_{i}, \dot{q}_{i}$ to $q_{f}, \dot{q}_{f}$. For given desired initial and final state values, virtual constraint can be easily deduced to join the desired values for the actuated and controlled variables. However, the evolution of the unactuated variables is known only by integration of the dynamics (15); a desirable dynamic behavior is imposed on these variables by the use of equality and inequality constraints in the optimization process.

\section{B. Specifics}

Here, Bezier polynomials of degree 3 are chosen to define the virtual constraints ${ }^{3}$. The virtual constraints are expressed as functions of the variable $\theta$; see (8). From $q_{i}$ and $q_{f}$, the initial and final values of $\theta$, denoted $\theta_{i}$ and $\theta_{f}$, can be calculated. Let

$$
h_{d}(\theta)=\sum_{k=0}^{3} \alpha_{k} \frac{3 !}{k !(3-k) !} s^{k}(1-s)^{3-k},
$$

where

$$
s=\frac{\theta-\theta_{i}}{\theta_{f}-\theta_{i}}
$$

is the normalized independent variable. The coefficients of the Bezier polynomials, $\alpha_{k}$, are $(6 \times 1)$ vectors of real numbers. They must be determined so as to join $\left(q_{i}\right)_{a}$ to $\left(q_{f}\right)_{a}$ and $\left(\dot{q}_{i}\right)_{a}$ to $\left(\dot{q}_{f}\right)_{a}$, (the additional subscript " $a$ " denotes the actuated variables) when $\theta$ varies from $\theta_{i}$ to $\theta_{f}$, yielding

$$
\begin{aligned}
\alpha_{0} & =h_{d}\left(\theta_{i}\right)=\left(q_{i}\right)_{a} \\
\alpha_{1} & =\left(q_{i}\right)_{a}+\frac{\theta_{f}-\theta_{i}}{3} \frac{\partial h_{d}}{\partial \theta}\left(\theta_{i}\right)=\left(q_{i}\right)_{a}+\frac{\theta_{f}-\theta_{i}}{3} \frac{\left(\dot{q}_{i}\right)_{a}}{\dot{\theta}_{i}} \\
\alpha_{2} & =\left(q_{f}\right)_{a}-\frac{\theta_{f}-\theta_{i}}{3} \frac{\partial h_{d}}{\partial \theta}\left(\theta_{f}\right)=\left(q_{f}\right)_{a}-\frac{\theta_{f}-\theta_{i}}{3} \frac{\left(\dot{q}_{f}\right)_{a}}{\dot{\theta}_{f}} \\
\alpha_{3} & =h_{d}\left(\theta_{f}\right)=\left(q_{f}\right)_{a} .
\end{aligned}
$$

The evolution of the unactuated variables is calculated by integration of the dynamic subsystem (15), that is, the stance phase zero dynamics, starting from the initial state $\left(q_{i}\right)_{u}=$ $\left[\left(q_{i}\right)_{1}, \theta_{i}\right]^{\prime}$ and terminating at $\theta=\theta_{f}$, where $\left(q_{i}\right)_{1}$ denotes the initial value of $q_{1}$ for the first step.

When the evolution of the unactuated variables is calculated, because the evolution of the actuated variable is given by (7) and (16), the required torque can be calculated by the second line of equation (13), and the ground reaction force $F_{s t}$ expressed in the inertial reference frame (see Fig. 1) can be calculated as well.

The search for a periodic walking motion can now be cast as a constrained nonlinear optimization problem: Find the 15 optimization parameters prescribing $\left(q_{f}, \dot{q}_{f}\right)$ that minimize the integral-squared torque per step length,

$$
J=\frac{1}{L} \int_{0}^{T} u^{*^{\prime}} u^{*} d t
$$

where $T$ is the walking period and $L$ is the step length, while satisfying symmetry (1), and subject to the following:

\section{inequality constraints}

\footnotetext{
${ }^{3}$ A degree greater than 3 can also be chosen, in which case the number of optimization variables increases [25].
}

- $\theta$ is strictly increasing (i.e, $\dot{\theta}>0$ along the solution);

- the swing foot is positioned above the ground $\left(z_{s w} \geq\right.$ $0)$;

- a no-take-off constraint, $F_{s t}(3)>0$;

- a friction constraint, $\sqrt{F_{s t}(1)^{2}+F_{s t}(2)^{2}} \leq \mu F_{s t}(3)$; equality constraints

and a set of conditions imposing periodicity,

$$
\begin{aligned}
q_{1}(T) & =\left(q_{f}\right)_{1} \\
\dot{q}_{1}(T) & =\left(\dot{q}_{f}\right)_{1} \\
\dot{\theta}(T) & =\dot{\theta}_{f},
\end{aligned}
$$

where $q_{1}(t)$ and $\theta(t)$ result from the integration of the zero dynamics and the walking period $T$ is such that $\theta(T)=\theta_{f}$.

The above procedure can be performed in MATLAB with the FMINCON function of the optimization toolbox. A fixedpoint solution $x^{*}=\left[q_{f}^{*}, \dot{q}_{f}^{*}\right]^{\prime}$ minimizing $J$ defines a desired periodic walking cycle (or nominal orbit). The criterion being optimized (19) has many local minima and the optimization technique used is local. Thus, the obtained optimal periodic motion depends on the initial set of optimization parameters.

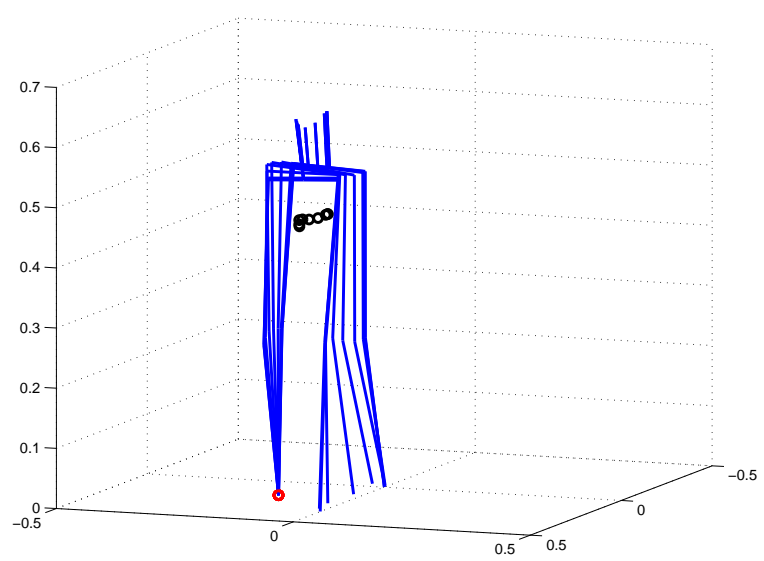

Fig. 4. Stick-diagram of the optimal motion with respect to the torque criterion.

\section{An example periodic motion minimizing integral-squared torque}

The physical parameters of the 3D biped studied here are given in Table I. For these parameters, a periodic orbit was computed following the technique presented in the previous subsection. We obtained a periodic motion defined by $x^{*}=$ $\left(q_{f}^{*}, \dot{q}_{f}^{*}\right)$, where

$$
\begin{gathered}
q_{f}^{*}=[-0.0174,-0.34038,0.3820,-0.2940,0.0602,0.0487, \\
-0.5077,0.1688]^{\prime}, \\
\dot{q}_{f}^{*}=[-0.4759,-1.1825,0.0997,0.2785,-0.1000,0.1000, \\
1.398,0]^{\prime} .
\end{gathered}
$$

A stick-figure diagram for the first step of the periodic walking gait is presented in Fig. 4. The walking gait has a period of $T=0.39$ seconds, a step size of $L=0.176 \mathrm{~m}$, and an average walking speed of $0.447 \mathrm{~m} / \mathrm{sec}$, or 0.745 body lengths per second. The step width is $0.156 \mathrm{~m}$, close to the hip width. The peak torque required to produce the periodic motion is less than $10 \mathrm{Nm}$ for each joint. 


\begin{tabular}{|l|l|l|l|l|l|l|l|}
\hline $\mathrm{g}$ & $\mathrm{W}$ & $\mathrm{L} 1$ & $\mathrm{~L} 2$ & $\mathrm{~L} 3$ & $\mathrm{~m} 1$ & $\mathrm{~m} 2$ & $\mathrm{~m} 3$ \\
\hline 9.81 & 0.15 & 0.275 & 0.275 & 0.05 & 0.875 & 0.875 & 5.5 \\
\hline
\end{tabular}

TABLE I

PARAMETERS FOR THE 3D BIPEDAL ROBOT (IN MKS).

\section{CReating a Hybrid Zero Dynamics}

The stability of a fixed-point $x^{*}$ can be tested numerically by linearizing the Poincare map about the fixed-point as presented in [19]. This numerical stability test has a high computational cost, however, because it requires the estimation of the Jacobian of the Poincaré map, in a space of dimension $2 n-1$, where $n$ is the number of independent joint coordinates; here $n=8$. We propose a slight modification of the control law in order to be able to study the stability of the closed-loop system in a reduced-dimensional state space.

\section{A. Hybrid zero dynamics (HZD) and a stability test in a reduced space}

The control law (10) is such that, on the periodic orbit, the virtual constraints (7) are identically satisfied. However, off the periodic orbit, even if the virtual constraints are satisfied at the end of given step, they will not in general be satisfied ${ }^{4}$ at the beginning of the next step. Consequently, the behavior of the robot cannot be deduced from the behavior of the uncontrolled variables $q_{u}$ and the simulation of the complete model is required to predict the behavior of the robot. In the language of [25], [27, Chap. 5], while the feedback control law (10) has created a zero dynamics of the stance phase dynamics, it has not created a hybrid zero dynamics, that is, a zero dynamics of the full hybrid model (6).

If the control law could be modified so as to create a hybrid zero dynamics, then the study of the swing phase zero dynamics (15) and the impact model would be sufficient to determine the stability of the complete closed-loop behavior of the robot, thereby leading to a reduced-dimension stability test. A modification of the control law to achieve a hybrid zero dynamics was first proposed in [17]; a second more easily implementable method has been given in [16], along with a complete stability analysis.

Following [16], the virtual constraints are modified stride to stride so that they are compatible with the initial state of the robot at the beginning of each step. The new output for the feedback control design is

$$
y_{c}=h\left(q, y_{i}, \dot{y}_{i}\right)=q_{a}-h_{d}(\theta)-h_{c}\left(\theta, y_{i}, \dot{y}_{i}\right) .
$$

This output consists of the previous output (7), and a correction term $h_{c}$ that depends on (7) evaluated at the beginning of the step, specifically, $y_{i}=q_{a, i}-h_{d}\left(\theta_{i}\right)$ and $\dot{y}_{i}=\dot{q}_{a, i}-\frac{\partial h_{d}(\theta)}{\partial \theta} \dot{\theta}_{i}$, where the subscript " $i$ " denotes the initial value for the current step. The values of $y_{i}, \dot{y}_{i}$ are updated at the beginning of each step and held constant

\footnotetext{
${ }^{4}$ This may be true for several reasons, one of which is that the virtual constraints may not have been chosen to be compatible with the impact map.
}

throughout the step. The function $h_{c}$ is taken to be a threetimes continuously differentiable function of $\theta$ such that ${ }^{5}$

$$
\left\{\begin{aligned}
h_{c}\left(\theta_{i}, y_{i}, \dot{y}_{i}\right) & =y_{i} \\
\frac{\partial h_{c}}{\partial \theta}\left(\theta_{i}\right) & =\dot{y}_{i} \\
h_{c}\left(\theta, y_{i}, \dot{y}_{i}\right) & \equiv 0, \quad \frac{\theta_{i}+\theta_{f}}{2} \leq \theta \leq \theta_{f}
\end{aligned}\right.
$$

With $h_{c}$ designed in this way, the initial errors of the output and its derivative are smoothly joined to the original virtual constraint at the middle of the step. In particular, for any initial error, the initial virtual constraint $h_{d}$ is exactly satisfied by the end of the step.

Under the new control law defined by (20), the behavior of the robot is completely defined by the impact map and the swing phase zero dynamics (15), where $h_{d}$ is replaced by $h_{d}+h_{c}$. The stability of a fixed-point $x^{*}$ can now be tested numerically using a restricted Poincaré map defined from $\rho: S \cap Z \rightarrow S \cap Z$, where $Z=\left\{(q, \dot{q}) \mid y_{c}(q)=\right.$ $\left.0, \dot{y}_{c}(q)=0\right\}$ and $S$ is the switching surface. The key point is that in $S \cap Z$, the state of the robot can be represented using only three independent variables, $x^{z}=\left[q_{1}, \dot{q}_{1}, \dot{\theta}\right]^{\prime}$, and hence the Jacobian of the restricted Poincaré map evaluated at a fixed point is a $3 \times 3$ matrix. From [16], for $\epsilon$ sufficiently small in (10), the exponential stability of a fixed-point of the restricted Poincaré map determines exponential stability of the full-order closed-loop robot model.

\section{B. Example of the periodic motion minimizing integral- squared torque}

We consider the virtual constraints corresponding to the optimal periodic motion obtained in Section V-C, and the control law defined in (20) is used.

To study the stability of this control law around the periodic motion, the eigenvalues of the linearized restricted Poincaré map are computed yielding

$$
\begin{aligned}
& \lambda_{1}=0.8878 \\
& \lambda_{2}=-0.6951 \\
& \lambda_{3}=-2.0891
\end{aligned}
$$

One eigenvalue has magnitude greater than one and hence the gait is unstable under this controller.

We have found that for most periodic motions optimized with respect to integral-squared torque per step length, (19), the obtained gait is unstable under the control law defined by (20). In the next section, freedom in the selection of the controlled outputs ${ }^{6}$ is used to obtain a stable walking cycle using only within-stride control.

\section{IMPROVED OUtPut SElEction}

In the previous sections, the controlled variables driven by the virtual constraints are simply the actuated variables,

${ }^{5}$ In our specific application, we used a fifth order polynomial for $\theta_{i} \leq$ $\theta \leq \frac{\theta_{i}+\theta_{f}}{2}$; continuity of position, velocity and acceleration is ensured at $\theta=\frac{\theta_{i}^{2}+\theta_{f}}{2}$.

${ }^{6}$ The controlled outputs are no longer the actuated variables as in (10), but a judiciously chosen linear combination of $q$. A convenient choice of outputs is given. 
$q_{a}$; see (7). The choice of the controlled variables directly affects the zero dynamics in (15). It is shown here that for the same desired periodic motion, the stability of the closed-loop system can be dramatically improved through a judicious choice of the controlled variables.

\section{A. Effect on the swing phase zero dynamics}

For simplicity, we limit our analysis to the case of controlled variables that are linear with respect to the configuration variables. Thus the controlled variables are

$$
q_{c}=M\left[\begin{array}{c}
q_{1} \\
\theta \\
q_{a}
\end{array}\right]=\left[\begin{array}{lll}
M_{1} & M_{\theta} & M_{a}
\end{array}\right]\left[\begin{array}{c}
q_{1} \\
\theta \\
q_{a}
\end{array}\right],
$$

where $M$ is a $(6 \times 8)$ constant matrix with $M_{a}$ invertible. A known periodic motion $q^{*}(t)$ can be reparameterized ${ }^{7}$ as function of the variable $\theta$, yielding $q^{*}(\theta)$. The virtual constraint for the new controlled variables then yields the output

$$
y=\underbrace{M\left[\begin{array}{c}
q_{1} \\
\theta \\
q_{a}
\end{array}\right]}_{h_{0}(q)}-\underbrace{M\left[\begin{array}{c}
q_{1}^{*}(\theta) \\
\theta \\
q_{a}^{*}(\theta)
\end{array}\right]}_{h_{d}(\theta)} .
$$

When the constraint is satisfied, $y \equiv 0$, equation (23) allows us to solve for $q_{a}$, giving

$$
q_{a}=q_{a}^{*}(\theta)+M_{a}^{-1} M_{1}\left(q_{1}^{*}(\theta)-q_{1}\right) .
$$

Substituting this equation into (14), we obtain for the swing phase zero dynamics

$$
\begin{gathered}
D_{11}\left(q_{u}\right)\left[\begin{array}{c}
\ddot{q}_{1} \\
\ddot{\theta}
\end{array}\right]+D_{12}\left(q_{u}\right)\left(\frac{\partial q_{a}^{*}}{\partial \theta} \ddot{\theta}+\frac{\partial^{2} q_{a}^{*}}{\partial \theta^{2}} \dot{\theta}^{2}\right)+ \\
D_{12}\left(q_{u}\right) M_{a}^{-1} M_{1}\left(\frac{\partial q_{1}^{*}}{\partial \theta} \ddot{\theta}+\frac{\partial^{2} q_{1}^{*}}{\partial \theta^{2}} \dot{\theta}^{2}-\ddot{q}_{1}\right)+H_{1}\left(q_{u}, \dot{q}_{u}\right)=0 .
\end{gathered}
$$

The nominal periodic motion satisfies both equations (25) and (15), but the two equations produce different solutions away from the periodic motion. When the principle of virtual constraints is applied to a system with only one degree of underactuation, namely $\theta$, which is common for example in planar bipeds, the swing phase zero dynamic is not affected by the choice of the output, and therefore the stability of a periodic orbit (i.e., walking motion) is not modified; only the transient motion can be different. In the case of a system with two degrees of underactuation, the choice of the controlled output can affect the stability of the gait via the choice of $M_{a}^{-1} M_{1}$.

In order to illustrate this property, a new choice of output is proposed. This choice is based on the following physical reasoning: The motion in the frontal direction is difficult to stabilize. The position of the center of mass in the frontal direction is important. If, at touchdown, the center of mass is not between the feet, but outside the position of the next supporting foot, the robot will topple sideways. Thus, the control of the variable $q_{6}$ (which regulates step width on the swing leg) is replaced by the control of the distance between

\footnotetext{
${ }^{7}$ This assumes that $\theta$ is monotonic.
}

the swing leg end and the center of mass along the frontal direction. To obtain a linear output, this function is linearized around the touchdown configuration to define $M$ in (23).

\section{B. Example of the periodic motion minimizing integral- squared torque}

The periodic motion described in Section $\mathrm{V}-\mathrm{C}$ can be stabilized using the new controlled output. As mentioned in the previous subsection, the actuated joints $q_{3}, q_{4}, q_{5}, q_{7}$, and $q_{8}$ are controlled via virtual constraints just as in the original control law. A new output $h_{d, 4}$ is considered, with this output no longer based on $q_{6}$ but instead on distance between the swing leg end and the center of mass along the frontal direction.

For this trajectory, for support on leg 1, the linearization around $q_{f}$ of the distance between the swing leg end and the center of mass along the frontal direction yields

$$
\begin{gathered}
d=-0.457 q_{1}-0.020 q_{2}-0.018 q_{3}-0.020 q_{4}-0.489 q_{5} \\
+0.461 q_{6}-0.056 q_{7}-0.022 q_{8} .
\end{gathered}
$$

On the periodic orbit, this distance is evaluated and approximated by a function of $\theta$, denoted $d^{*}(\theta)$. The new controlled output is then

$$
\begin{gathered}
y_{4}=-0.457 q_{1}-0.020 q_{2}-0.018 q_{3}-0.020 q_{4}-0.489 q_{5} \\
+0.461 q_{6}-0.056 q_{7}-0.022 q_{8}-d^{*}(\theta) .
\end{gathered}
$$

When the control law is defined using this new output, the walking gait is stable, as can be shown via the calculation of the eigenvalues of the linearization of the restricted Poincaré map:

$$
\begin{aligned}
\lambda_{1} & =0.7846 \\
\lambda_{2,3} & =-0.028 \pm 0.250 i \\
\left|\lambda_{2,3}\right| & =0.2512 .
\end{aligned}
$$

To illustrate the orbit's local exponential stability, the 3D biped's model in closed-loop is simulated with the initial state perturbed from the fixed-point $x^{*}$. An initial error of $-1^{\circ}$ is introduced on each joint and a velocity error of $-5^{\circ} s^{-1}$ is introduced on each joint velocity. Fig. 5 shows phase-plane plots of the first four variables. The convergence towards a periodic motion is clear for the controlled and uncontrolled variables.

\section{CONCLUSIONS}

An underactuated 3D bipedal model has been studied, with the objective of developing a time-invariant feedback control law that induces asymptotically stable walking, without relying on the use of large feet. The method of virtual constraints and hybrid zero dynamics was applied to the 3D robot, with the virtual constraints chosen via optimization as suggested in [26]. The main contributions of the paper are:

1) The computation of human-like periodic walking motions that can be stable or unstable, depending on the choice of actuated variables and corresponding virtual constraints. 

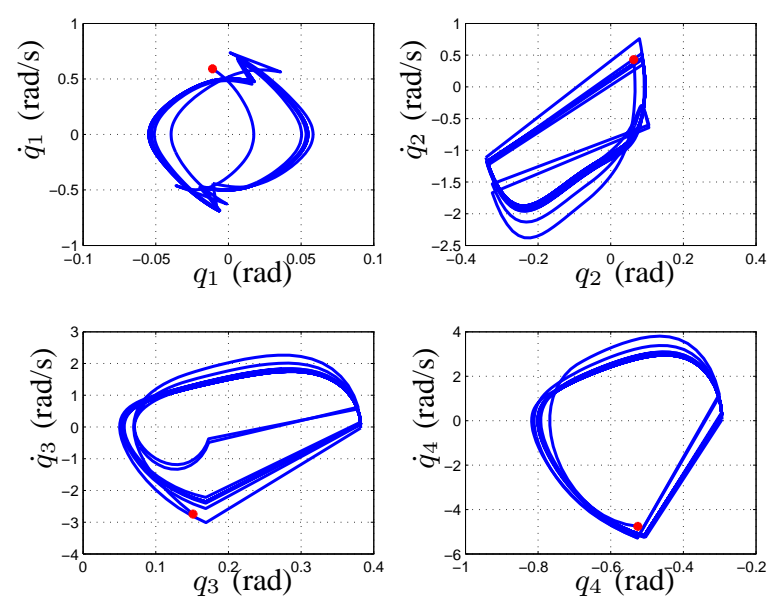

Fig. 5. Phase-plane plots for $q_{i}, i=1, \ldots, 4$. The straight lines correspond to the impact phase, where the state of the robot changes instantaneously. The initial state is represented by a (red) star. Each variable converges to a periodic motion

2) The numerical study of stability on the basis of a lowdimensional subsystem corresponding to the hybrid zero dynamics. The Poincaré return map was computed in a space of dimension three for a robot with two degrees of underactuation.

3) The discovery of the importance of the selection of the controlled outputs on the stability of a given periodic motion.

When the method of virtual constraints is applied to a bipedal model with only one degree of underactuation, which is common for example in planar bipeds, the swing phase zero dynamic is not affected by the choice of the output, and therefore the stability of a periodic orbit (i.e., walking motion) is unaffected by the choice of the controlled output; only the transient motion can be different. In the case of a biped with two degrees of underactuation, as in an underactuated 3D biped, the choice of the controlled output can affect the stability of the hybrid zero dynamics. A more systematic study of this property is needed.

ACKnowledgment: The work of J.W. Grizzle is supported by NSF grant ECS-0600869. The work of C. Chevallereau is supported by ANR grants for the PHEMA project. The work of C.L. Shih is supported by Taiwan NSC grant NSC-96-2221-E-011-126.

\section{REFERENCES}

[1] A. Ames and R. Gregg, "Stably extending two-dimensional bipedal walking to three," in Proc. of the 2007 American Control Conferenc, New York, NY, 2007, pp. 177-182.

[2] C. Chevallereau, G. Abba, Y. Aoustin, E. Plestan, F. Westervelt, C. Canduas-de Wit, and J. Grizzle, "RABBIT: A testbed for advanced control theory," IEEE Control Systems Magazine, vol. 23, no. 5, pp. 57-79, October 2003.

[3] C. Chevallereau, J. Grizzle, and C. Shih, "Asymptotically stable walking of a five-link underactuated 3d bipedal robot," Submitted to IEEE Trans. on Robotics, January 2008.

[4] J. H. Choi and J. Grizzle, "Planar bipedal walking with foot rotation," in Proc. of the American Control Conference, 2005, pp. 4909-16.

[5] S. Collins, A. Ruina, R. Tedrake, and M. Wisse, "Efficient bipedal robots based on passive-dynamic walkers," Science, no. 307, pp. 1082 $1085,2005$.
[6] T. Fukuda, M. Doi, Y. Hasegawa, and H. Kajima, Fast motions in Biomechanics and Robotics, ser. Lecture Notes in Control and Information Sciences. Heidelberg, Allemagne: Springer, 2006, ch. Multi-Locomotion Control of Biped Locomotion and Brachiation Robot, pp. 121-145.

[7] R. Gregg and M. Spong, "Reduction-based control with application to three-dimensional bipedal walking robots," in Pre-print, October 2007

[8] J. Grizzle, "Publications on robotics and control," www.eecs.umich.edu/ grizzle/papers/robotics.html, January 2008.

[9] J. Grizzle, G. Abba, and F. Plestan, "Asymptotically stable walking for biped robots: Analysis via systems with impulse effects," IEEE Transactions on Automatic Control, vol. 46, pp. 51-64, January 2001

[10] A. Isidori, Nonlinear Control Systems, 3rd ed. Berlin: SpringerVerlag, 1995.

[11] _ - Nonlinear Control Systems: An Introduction, 3rd ed. Berlin: Springer-Verlag, 1995.

[12] S. Kajita, F. Kanehiro, K. Kaneko, K. Fujiwara, K. Harada, K. Yokoi, and $\mathrm{H}$. Hirukawa, "Biped walking pattern generator allowing auxiliary zmp control," in Proceedings of the 2006 IEEE/RSJ International Conference on Intelligent Robots and Systems, 2006, pp. 2993-2999.

[13] S. Kajita, M. Morisawa, K. Harada, K. Kaneko, F. Kanehiro, K. Fujiwara, and $\mathrm{H}$. Hirukawa, "Biped walking pattern generation by using preview control of zero-moment point," in Proceedings of the ICRA '03 IEEE International Conference on Robotics and Automation, vol. 2, 2003, pp. 1620-1626.

[14] A. Kuo, "Stabilization of lateral motion in passive dynamic walking," International Journal of Robotics Research, vol. 18, no. 9, pp. 917 930, 1999.

[15] B. Morris and J. Grizzle, "A restricted Poincaré map for determining exponentially stable periodic orbits in systems with impulse effects: Application to bipedal robots," in IEEE Conf. on Decision and Control. Seville, Spain: IEEE Press, December 2005.

[16] _ - "Hybrid invariant manifolds in systems with impulse effects with application to periodic locomotion in bipedal robots," IEEE Transactions on Automatic Control, vol. pre-print, February 2007, (To appear).

[17] B. Morris, E. Westervelt, C. Chevallereau, G. Buche, and J. Grizzle, Fast Motions Symposium on Biomechanics and Robotics, ser. Lecture Notes in Control and Information Sciences. Heidelberg, Germany: Springer-Verlag, 2006, ch. Achieving Bipedal Running with RABBIT: Six Steps toward Infinity, pp. 277-97.

[18] F. Plestan, J. Grizzle, E. Westervelt, and G. Abba, "Stable walking of a 7-DOF biped robot," IEEE Transactions on Robotics and Automation, vol. 19, no. 4, pp. 653-668, August 2003.

[19] C. Shih, J. Grizzle, and C. Chevallereau, "Asymptotically stable walking of a simple underactuated 3d bipedal robot," in The 33rd Annual Conference of the IEEE Industrial Electronics Society (IECON), Taipei, Taiwan, Novembre 2007, pp. 2766-2771.

[20] G. Song and M. Zefran, "Underactuated dynamic three-dimensional bipedal walking," in Proceedings of the IEEE International Conference On Robotics and Automation. Orlando, Florida: IEEE Press, May 2006, pp. 854-859.

[21] M. Spong, "Energy based control of a class of underactuated mechanical systems," in Proc. of IFAC World Congress, San Francisco, CA, 1996, pp. 431-435.

[22] M. Spong and F. Bullo, "Controlled symmetries and passive walking,," IEEE Transactions on Automatic Control, vol. 50, no. 7, pp. 10251031, July 2005.

[23] A. Takanishi, Y. Egusa, M. Tochizawa, T. Takeya, and I. Kato, "Realisation of dynamic walking stabilized with trunk motion," in Proceedings of ROMANSY 7, 1988, pp. 68-79.

[24] M. Vukobratović, B. Borovac, and V. Potkonjak, "ZMP: A review of some basic misunderstandings," International Journal of Humanoid Robotics, vol. 3, no. 2, pp. 153-75, June 2006.

[25] E. Westervelt, J. Grizzle, and D. Koditschek, "Zero dynamics of planar biped walkers with one degree of under actuation," in IFAC 2002, Bareclona, Spain, July 2002.

[26] — - "Hybrid zero dynamics of planar biped walkers," IEEE Transactions on Automatic Control, vol. 48, no. 1, pp. 42-56, January 2003

[27] E. Westervelt, J. Grizzle, C. Chevallereau, J. Choi, and B. Morris, Feedback Control of Dynamic Bipedal Robot Locomotion, ser. Control and Automation. Boca Raton: CRC Press, June 2007. 